\title{
Monotone interval fuzzy neutrosophic soft eigenproblem
}

\author{
P. Murugadas ${ }^{1 *}$, M. Kavitha ${ }^{2}$ and S. Sriram ${ }^{3}$
}

\begin{abstract}
The Interval Fuzzy Neutrosophic Soft Eigenproblem in max-min Fuzzy Neutrosopic Soft Algebra (FNSA) is studied. A classification of Interval Fuzzy neutrosophic Soft Eigenvectors (IFNSEvs) is introduced and six types of IFNSEvs are described. Characterization of all six types is given for the case of strictly increasing FNSEvs and Hasse diagram of relations between the types are presented.
\end{abstract}

Keywords

Fuzzy Neutrosophic Soft Set (FNSS), Fuzzy Neutrosophic Soft Matrices(FNSMs), Fuzzy Neutrosophic Soft Eigenvector (FNSEvs), Interval Fuzzy Neutrosophic Soft Eigenvectors (IFNSEvs), Interval Fuzzy Neutrosophic Soft Matrices (IFNSMs) AMS Subject Classification

Primary 03E72; Secondary 15B15.

${ }^{1}$ Department of Mathematics, Government Arts and Science College, Karur-639 005, India.

${ }^{2}$ Department of Mathematics, Annamalai University, Annamalainagar-608002, India.

${ }^{3}$ Mathematics wing, Directorate of Distance Education, Annamalai University, Annamalainagar-608002, Tamil Nadu, India.

*Corresponding author: ${ }^{*}$ bodi_muruga@yahoo.com; ${ }^{2}$ kavithakathir3@gmail.com; ${ }^{3}$ ssm_3096@yahoo.co.in

Article History: Received 24 March 2019; Accepted 09 May 2019

(C)2019 MJM

\section{Contents}

1 Introduction 342

2

Preliminaries ............................. 343

3 Interval Fuzzy Neutrosophic Soft Eigenvectors Classification 344

4 Relations between various types of monotone FNSEvs 347

5 Conclusion 349

References 349

\section{Introduction}

Most of our traditional tools for formal modeling, reasoning and computing are crisp, deterministic and precise in character. However, in real life, there are many complicated problems in Engineering, Economics, Environment, Social Sciences, Medical Sciences etc., that involve data which are not all always crisp, precise and deterministic in character because of various uncertainties of typical problems. Such uncertainties are being dealt with the help of the theories, like theory of Probability, theory of Fuzzy sets (Zadeh 1965) [38], theory of Intuitionistic fuzzy sets (Atanassov 1986)[3], theory of Vague set (Gau and Buehrer 1993) [5, 13], theory of
Interval Mathematics (Moore, 1996) [26, 33] and theory of Rough sets (Pawlak 1982) [27]. But all these theories have their own difficulties. The reason of the difficulties is possibly, the inadequacy of the parametrization tool of the theories.

To overcome these difficulties Molodtsov [29] introduced the concept of soft set as a new mathematical tool for dealing with uncertainty which is free from the difficulties that have troubled the usual theoretical approaches. He successfully applied the soft theory into several directions, such as smoothness of functions, game theory, operations research, Riemann integration, Perron integration, theory of probability, theory of measurement and so on. Maji et al. [22] initiated the concept of soft sets with some properties regarding fuzzy soft union, intersection, complement of fuzzy soft set and they are applied in decision making problem. Further, [24] Maji et. al, successfully extended the soft set as fuzzy soft set and intuitionistic fuzzy soft set and studied the application of these soft sets in decision-making problems. Jang et. al, studied interval valued intuitionistic fuzzy sets.

The IFS can only handle the incomplete information considering both the truth-membership (or simply membership) and falsity-membership (or non-membership) values. It does not handle the indeterminate and inconsistent information which exists in belief system. Smarandache [32] introduced 
the concept of neutrosophic set which is a mathematical tool for handling problems involving imprecise, indeterminacy and inconsistent data. This theory is a powerful tool which generalize the concept of the classical set, fuzzy set, intuitionistic fuzzy set, paraconsistent sets, dialetheist sets, paradoxist sets, and tautological set and so on.

Fuzzy matrices defined first time by Thomason in 1977 [34] and discussed about the convergence of the powers of a fuzzy matrix. The theory of fuzzy matrices were developed by Kim and Roush [20] as an extension of Boolean matrices. Manoj Bora et al. [28] have applied intuitionistic fuzzy soft matrices in the medical diagnosis problem. Arockiarani and Sumathi [1, 2, 36] introduced Fuzzy Neutrosophic Soft Matrix (FNSM) and used them in decision making problems also in which they defined new type of operations. Broumi et al. [4] proposed the concept of generalized interval neutrosophic soft set and studied their operations. Also, they presented an application of it in decision making problem. Kavitha et al. [16-18] introduced the concept of unique solvability of max-min operation through FNSM equation $A x=b$ and explained strong regularity of FNSMs over fuzzy neutrosophic soft algebra and computing the greatest X-eigenvector of fuzzy neutrosophic soft matrix. They also addressed on the power of fuzzy neutrosophic soft matrix. In [37], Uma et. al, introduced the concept of fuzzy neutrosophic soft matrices of Type- 1 and Type- 2 .

In practice, the values of vector or matrix inputs are not exact numbers and often they are rather contained in some intervals. Considering matrices and vectors with interval coefficients is therefore of great practical importance, see [7, 8, 10, 14, 30]. This paper investigates monotone IFNSEvs of IFNSMs in max-min FNSA.

By max-min FNSA we understand a triple $(\mathscr{N}, \oplus, \otimes)$, where $\mathscr{N}$ is a linearly ordered set and $\oplus=\max , \otimes=\min$ are binary operations on $\mathscr{N}$. The notation $\mathscr{N}_{(m, n)}, \mathscr{N}_{(n)}$ denotes the set of all FNSMs, FNSVs of given dimension over $\mathscr{N}$. Operations $\otimes, \otimes$ are extended to FNSMs and FNSVs in a formal way. The linear ordering on $\mathscr{N}$ induces partial ordering on $\mathscr{N}_{(m, n)}$ and $\mathscr{N}_{(n)}$, the notations $\wedge(\vee)$ and $\Lambda(\vee)$ are used for the operation of meet (join) in these sets.

The FNSE problem for a given FNSM $A \in \mathscr{N}_{(n, n)}$ in maxmin FNSA consists of finding a value $\left\langle\lambda^{T}, \lambda^{I}, \lambda^{F}\right\rangle \in \mathscr{N}$ (FNSE value) and a FNSV $\left\langle x^{T}, x^{I}, x^{F}\right\rangle \in \mathscr{N}_{(n)}$ (FNSEv) such that the equation

$A \otimes\left\langle x^{T}, x^{I}, x^{F}\right\rangle=\left\langle\lambda^{T}, \lambda^{I}, \lambda^{F}\right\rangle \otimes\left\langle x^{T}, x^{I}, x^{F}\right\rangle$ holds true. It is well-known that the above problem in max-min FNSA can be reduced to solving the equation. $A \otimes\left\langle x^{T}, x^{I}, x^{F}\right\rangle=\left\langle x^{T}, x^{I}, x^{F}\right\rangle$. Th FNSE problem in max-min FNSA has been studied by many authors. Interesting results were found in describing the structure of the FNSE space (the set of all FNSEvs), and algorithms for computing the largest FNSEv of a given FNSM were suggested in [18].

A classification consisting of six different types of IFNSEvs in presented in this paper and detailed characterization of all described types is given for strictly increasing IFNSEvs using the methods provided in [11].

\section{Preliminaries}

This section basically describes Neutrosophic Set (NS), Fuzzy Neutrosophic Soft Set (FNSS), Fuzzy Neutrosophic Soft Matrix (FNSM) and Fuzzy Neutrosophic Soft Matrices of type-I.

Definition 2.1. [35] A neutrosophic set $A$ on the universe of discourse $X$ is defined as $A=\left\{\left\langle x, T_{A}(x), I_{A}(x), F_{A}(x)\right\rangle, x \in\right.$ $X\}$, where $T, I, F: X \rightarrow]^{-} 0,1^{+}[$and

$$
{ }^{-} 0 \leq T_{A}(x)+I_{A}(x)+F_{A}(x) \leq 3^{+} .
$$

From philosophical point of view the neutrosophic set takes the value from real standard or non-standard subsets of ]$^{-} 0,1^{+}$, But in real life application especially in Scientific and Engineering problems it is difficult to use neutrosophic set with value from real standard or non-standard subset of ]$^{-} 0,1^{+}[$. Hence we consider the neutrosophic set which takes the value from the subset of $[0,1]$. Therefore we can rewrite equation (2.1) as $0 \leq T_{A}(x)+I_{A}(x)+F_{A}(x) \leq 3$. In short an element $\widetilde{a}$ in the neutrosophic set $A$, can be written as $\tilde{a}=\left\langle a^{T}, a^{I}, a^{F}\right\rangle$, where $a^{T}$ denotes degree of truth, $a^{I}$ denotes degree of indeterminacy, $a^{F}$ denotes degree of falsity such that $0 \leq a^{T}+a^{I}+a^{F} \leq 3$.

Example 2.2. Assume that the universe of discourse $X=$ $\left\{x_{1}, x_{2}, x_{3}\right\}$ where $x_{1}, x_{2}$ and $x_{3}$ characterize the quality, reliability, and the price of the objects. It may be further assumed that the values of $\left\{x_{1}, x_{2}, x_{3}\right\}$ are in $[0,1]$ and they are obtained from some investigations of some experts. The experts may impose their opinion in three components viz; the degree of goodness, the degree of indeterminacy and the degree of poorness to explain the characteristics of the objects. Suppose A is a Neutrosophic Set (NS) of $X$, such that $A=\left\{\left\langle x_{1}, 0.4,0.5,0.3\right\rangle,\left\langle x_{2}, 0.7,0.2,0.4\right\rangle\right.$,

$\left.\left\langle x_{3}, 0.8,0.3,0.4\right\rangle\right\}$ where for $x_{1}$ the degree of goodness of quality is 0.4 , degree of indeterminacy of quality is 0.5 and degree of falsity of quality is 0.3 etc,.

Definition 2.3. [29] Let $U$ be the initial universe set and $E$ be a set of parameter. Consider a non-empty set $A, A \subset E$. Let $P(U)$ denotes the set of all fuzzy neutrosophic sets of $U$. The collection $(F, A)$ is termed to the fuzzy neutrosophic soft set over $U$, where $F$ is a mapping given by $F: A \rightarrow P(U)$. Here after we simply consider $A$ as FNSS over $U$ instead of $(F, A)$.

Definition 2.4. [2] Let $U=\left\{c_{1}, c_{2}, \ldots, c_{m}\right\}$ be the universal set and $E$ be the set of parameters given by $E=\left\{e_{1}, e_{2}, \ldots, e_{m}\right\}$. Let $A \subset E$. A pair $(F, A)$ be a FNSS over $U$. Then the subset of $U \times E$ is defined by $R_{A}=\left\{(u, e) ; e \in A, u \in F_{A}(e)\right\}$ which is called a relation form of $\left(F_{A}, E\right)$. The membership function, indeterminacy membership function and non membership function are written by

$T_{R_{A}}: U \times E \rightarrow[0,1], I_{R_{A}}: U \times E \rightarrow[0,1]$ and $F_{R_{A}}: U \times E \rightarrow$ $[0,1]$ where $T_{R_{A}}(u, e) \in[0,1], I_{R_{A}}(u, e) \in[0,1]$ and $F_{R_{A}}(u, e) \in$ 
$[0,1]$ are the membership value, indeterminacy value and non membership value respectively of $u \in U$ for each $e \in E$.

If $\left[\left(T_{i j}, I_{i j}, F_{i j}\right)\right]=\left[T_{i j}\left(u_{i}, e_{j}\right), I_{i j}\left(u_{i}, e_{j}\right), F_{i j}\left(u_{i}, e_{j}\right)\right]$ we de-

$\left[\left\langle T_{i j}, I_{i j}, F_{i j}\right\rangle\right]_{m \times n}=\left[\begin{array}{ccc}\left\langle T_{11}, I_{11}, F_{11}\right\rangle & \ldots & \left\langle T_{1 n}, I_{1 n}, F_{1 n}\right\rangle \\ \left\langle T_{21}, I_{21}, F_{21}\right\rangle & \ldots & \left\langle T_{2 n}, I_{2 n}, F_{2 n}\right\rangle \\ \vdots & \vdots & \vdots \\ \left\langle T_{m 1}, I_{m 1}, F_{m 1}\right\rangle & \ldots & \left\langle T_{m n}, I_{m n}, F_{m n}\right\rangle\end{array}\right]$

Which is called an $m \times n F N S M$ of the FNSS $\left(F_{A}, E\right)$ over $U$

Definition 2.5. [37] Let $A=\left(\left\langle a_{i j}^{T}, a_{i j}^{I}, a_{i j}^{F}\right\rangle\right)$,

$B=\left\langle\left(b_{i j}^{T}, b_{i j}^{I}, b_{i j}^{F}\right)\right\rangle \in \mathscr{N}_{m \times n}$. The component wise addition and component wise multiplication is defined as

$A \oplus B=\left(\sup \left\{a_{i j}^{T}, b_{i j}^{T}\right\}, \quad \sup \left\{a_{i j}^{I}, b_{i j}^{I}\right\}, \quad \inf \left\{a_{i j}^{F}, b_{i j}^{F}\right\}\right)$ $A \otimes B=\left(\inf \left\{a_{i j}^{T}, b_{i j}^{T}\right\}, \quad \inf \left\{a_{i j}^{I}, b_{i j}^{I}\right\}, \quad \sup \left\{a_{i j}^{F}, b_{i j}^{F}\right\}\right)$

Definition 2.6. Let $A \in \mathscr{N}_{m \times n}, B \in \mathscr{N}_{n \times p}$, the composition of $A$ and $B$ is defined as.

$$
\begin{aligned}
A \circ B= & \left(\sum_{k=1}^{n}\left(a_{i k}^{T} \wedge b_{k j}^{T}\right), \quad \sum_{k=1}^{n}\left(a_{i k}^{I} \wedge b_{k j}^{I}\right), \prod_{k=1}^{n}\left(a_{i k}^{F} \vee b_{k j}^{F}\right)\right) \\
& \text { equivalently we can write the same as } \\
= & \left(\bigvee_{k=1}^{n}\left(a_{i k}^{T} \wedge b_{k j}^{T}\right), \bigvee_{k=1}^{n}\left(a_{i k}^{I} \wedge b_{k j}^{I}\right), \quad \bigwedge_{k=1}^{n}\left(a_{i k}^{F} \vee b_{k j}^{F}\right)\right) .
\end{aligned}
$$

The product $A \circ B$ is defined if and only if the number of columns of $A$ is same as the number of rows of $B$. Then $A$ and $B$ are said to be conformable for multiplication. We shall use $A B$ instead of $A \circ B$. Where $\sum\left(a_{i k}^{T} \wedge b_{k j}^{T}\right)$ means max-min operation and $\prod_{k=1}^{n}\left(a_{i k}^{F} \vee b_{k j}^{F}\right)$ means min-max operation.

\section{Interval Fuzzy Neutrosophic Soft Eigenvectors Classification}

In this section we define six types of IFNSEvs of IFNSMs and describe the necessary and sufficient conditions for these types of monotone IFNSEvs.

Let $n$ be a given natural number. We shall use the notation $N=\{1,2, \ldots, n\}$. Similarly to $[8,10,14]$, we define IFNSM with bounds $\underline{A}, \bar{A} \in \mathscr{N}_{(n, n)}$ and IFNSV with bounds $\left\langle\underline{x}^{T}, \underline{x}^{I}, \underline{x}^{F}\right\rangle,\left\langle\bar{x}^{T}, \bar{x}^{I}, \bar{x}^{F}\right\rangle \in \mathscr{N}_{(n)}$, as follows

$[\underline{A}, \bar{A}]=\left\{A \in \mathscr{N}_{(n, n)} ; \underline{A} \leq A \leq \bar{A}\right\}$,

$\left[\left\langle\underline{x}^{T}, \underline{x}^{I}, \underline{x}^{F}\right\rangle,\left\langle\bar{x}^{T}, \bar{x}^{I}, \bar{x}^{F}\right\rangle\right]=\left\{\left\langle x^{T}, x^{I}, x^{F}\right\rangle \in \mathscr{N}_{(n)} ;\left\langle\underline{x}^{T}, \underline{x}^{I}, \underline{x}^{F}\right\rangle \leq\right.$ $\left.\left\langle x^{T}, x^{I}, x^{F}\right\rangle \leq\left\langle\bar{x}^{T}, \bar{x}^{I}, \bar{x}^{F}\right\rangle\right\}$.

We assume in this section that an IFNSM $\mathbf{A}=[\underline{A}, \bar{A}]$ and an IFNSEv

$\mathbf{X}=\left[\left\langle\underline{x}^{T}, \underline{x}^{I}, \underline{x}^{F}\right\rangle,\left\langle\bar{x}^{T}, \bar{x}^{I}, \bar{x}^{F}\right\rangle\right]$ are fixed. The IFNSE problem for $\mathbf{A}$ and $\mathbf{X}$ consists in recognizing whether $A \otimes\left\langle x^{T}, x^{I}, x^{F}\right\rangle=$ $\left\langle x^{T}, x^{I}, x^{F}\right\rangle$

holds true for $A \in \mathbf{A},\left\langle x^{T}, x^{I}, x^{F}\right\rangle \in \mathbf{X}$. In dependence on the applied quantifiers, we get six types of IFNSEvs.

Definition 3.1. If IFNSM A is given, then IFNSV $\mathbf{X}$ is called 1. Strong FNSEv of $\mathbf{A}$ if $(\forall A \in \mathbf{A})\left(\forall\left\langle x^{T}, x^{I}, x^{F}\right\rangle \in \mathbf{X}\right)$

$\left[A \otimes\left\langle x^{T}, x^{I}, x^{F}\right\rangle=\left\langle x^{T}, x^{I}, x^{F}\right\rangle\right]$,

2. Strong universal FNSEv of $\mathbf{A}$ if

$\left(\exists\left\langle x^{T}, x^{I}, x^{F}\right\rangle \in \mathbf{X}\right)(\forall A \in \mathbf{A})\left[A \otimes\left\langle x^{T}, x^{I}, x^{F}\right\rangle=\left\langle x^{T}, x^{I}, x^{F}\right\rangle\right]$,

3. Universal FNSEv of $\mathbf{A}$ if

$(\forall A \in \mathbf{A})\left(\exists\left\langle x^{T}, x^{I}, x^{F}\right\rangle \in \mathbf{X}\right)\left[A \otimes\left\langle x^{T}, x^{I}, x^{F}\right\rangle=\left\langle x^{T}, x^{I}, x^{F}\right\rangle\right]$,

4. Strong tolerance FNSEv of $\mathbf{A}$ if

$(\exists A \in \mathbf{A})\left(\forall\left\langle x^{T}, x^{I}, x^{F}\right\rangle \in \mathbf{X}\right)\left[A \otimes\left\langle x^{T}, x^{I}, x^{F}\right\rangle=\left\langle x^{T}, x^{I}, x^{F}\right\rangle\right]$,

5. Tolerance FNSEv of $\mathbf{A}$ if

$\left(\forall\left\langle x^{T}, x^{I}, x^{F}\right\rangle \in \mathbf{X}\right)(\exists A \in \mathbf{A})\left[A \otimes\left\langle x^{T}, x^{I}, x^{F}\right\rangle=\left\langle x^{T}, x^{I}, x^{F}\right\rangle\right]$, 6. Weak FNSEv of $\mathbf{A}$ if

$(\exists A \in \mathbf{A})\left(\exists\left\langle x^{T}, x^{I}, x^{F}\right\rangle \in \mathbf{X}\right)\left[A \otimes\left\langle x^{T}, x^{I}, x^{F}\right\rangle=\left\langle x^{T}, x^{I}, x^{F}\right\rangle\right]$. Analogously as in [11], we denote the set of all strictly increasing FNSVs of dimension $n$ as

$$
\begin{gathered}
\mathscr{N}_{(n)}^{<}=\left\{\left\langle x^{T}, x^{I}, x^{F}\right\rangle \in \mathscr{N}_{(n)} ;(\forall i, j, \in N)[i \leq j \Rightarrow\right. \\
\left.\left.\left\langle x_{i}^{T}, x_{i}^{I}, x_{i}^{F}\right\rangle<\left\langle x_{j}^{T}, x_{j}^{I}, x_{j}^{F}\right\rangle\right]\right\},
\end{gathered}
$$

and the set of all increasing FNSV as

$$
\begin{gathered}
\mathscr{N}_{(n)}^{\leq}=\left\{\left\langle x^{T}, x^{I}, x^{F}\right\rangle \in \mathscr{N}_{(n)} ;(\forall i, j, \in N)[i<j \Rightarrow\right. \\
\left.\left.\left\langle x_{i}^{T}, x_{i}^{I}, x_{i}^{F}\right\rangle \leq\left\langle x_{j}^{T}, x_{j}^{I}, x_{j}^{F}\right\rangle\right]\right\} .
\end{gathered}
$$

Further we denote the FNSE space of a FNSM $A \in \mathscr{N}_{(n, n)}$ as $\mathscr{F}(A)=\left\{\left\langle x^{T}, x^{I}, x^{F}\right\rangle \in \mathscr{N}_{(n)} ; A \otimes\left\langle x^{T}, x^{I}, x^{F}\right\rangle=\left\langle x^{T}, x^{I}, x^{F}\right\rangle\right\}$, and the FNSE space of all strictly increasing FNSEvs (increasing FNSEvs) as

$$
\mathscr{F}<(A)=\mathscr{F}(A) \cap \mathscr{N}_{(n)}^{<}, \mathscr{F} \leq(A)=\mathscr{F}(A) \cap \mathscr{N}_{(n)}^{\leq} .
$$

it is clear that any FNSV $\left\langle x^{T}, x^{I}, x^{F}\right\rangle \in \mathscr{N}_{(n)}$ can be permuted to an increasing FNSV. Therefore, in view of the next theorem, the structure of the FNSE space $\mathscr{F}(A)$ of a given $n \times n$ maxmin FNSM $A$ can be described by investigating the structure of monotone FNSE spaces $\mathscr{F}<\left(A_{\varphi \varphi}\right)$ and $\mathscr{F} \leq\left(A_{\varphi \varphi}\right)$, for all permutations $\varphi$ on $N$.

Theorem 3.2. Let $A \in \mathscr{N}_{(n, n)},\left\langle x^{T}, x^{I}, x^{F}\right\rangle \in \mathscr{N}_{(n)}$ and let $\varphi$ be a permutation on $N$. Then $\left\langle x^{T}, x^{I}, x^{F}\right\rangle \in \mathscr{F}(A)$ if and only if $\left\langle x^{T}, x^{I}, x^{F}\right\rangle_{\varphi} \in \mathscr{F}\left(A_{\varphi \varphi}\right)$.

Proof. Let $\varepsilon$ be the identical permutation on $N$. It is easy to see that the following formulas are equivalent:

$A \otimes\left\langle x^{T}, x^{I}, x^{F}\right\rangle=\left\langle x^{T}, x^{I}, x^{F}\right\rangle, A_{\varphi \varepsilon} \otimes\left\langle x^{T}, x^{I}, x^{F}\right\rangle=\left\langle x^{T}, x^{I}, x^{F}\right\rangle_{\varphi}$, $A_{\varphi \varphi} \otimes\left\langle x^{T}, x^{I}, x^{F}\right\rangle_{\varphi}=\left\langle x^{T}, x^{I}, x^{F}\right\rangle_{\varphi}$. By this, the proof is complete.

For $A \in \mathscr{N}_{(n, n)}$ the structure of $\mathscr{F}<(A)$ has been described in [11] as an interval of strictly increasing FNSVs. FNSVs $m^{*}(A), M^{*}(A) \in \mathscr{N}_{(n)}$ are defined as follows.

For any $i \in N$, we put

$$
\begin{aligned}
m_{i}^{*}(A):= & \max _{j \leq i} \max _{k>j}\left\langle a_{j k}^{T}, a_{j k}^{I}, a_{j k}^{F}\right\rangle, M_{i}^{*}(A):= \\
& \min _{j \geq i} \max _{k \geq j}\left\langle a_{j k}^{T}, a_{j k}^{I}, a_{j k}^{F}\right\rangle .
\end{aligned}
$$

Remark 3.3. If a maximum of an empty set should be computed in the above definition of $m^{*}(A)$, then we use the fact that, by usual definition, max $\phi$ is the least element in $\mathscr{N}$. 
Theorem 3.4. [11] Let $A \in \mathscr{N}_{(n, n)}$ and $\left\langle x^{T}, x^{I}, x^{F}\right\rangle \in \mathscr{N}_{(n)}$ be a strictly increasing FNSV. Then $\left\langle x^{T}, x^{I}, x^{F}\right\rangle \in \mathscr{F}(A)$ if and only if $m^{*}(A) \leq\left\langle x^{T}, x^{I}, x^{F}\right\rangle \leq M^{*}(A)$. In formal notation,

$$
\mathscr{F}^{<}(A)=\left\langle m^{*}(A), M^{*}(A)\right\rangle \cap \mathscr{N}_{(n)}^{<} .
$$

In this paper our considerations will be restricted to strictly increasing FNSEvs in $\mathbf{X}$. The restricted IFNSEvs will be called monotone IFNSEvs and similarly, the name of the restricted types will be extended by 'monotone', i.e. monotone strong FNSEv, ,...,monotone weak FNSEv.

Formally, we denote by $\mathbf{X}^{<}=\left[\left\langle\underline{x}^{T}, \underline{x}^{I}, \underline{x}^{F}\right\rangle,\left\langle\bar{x}^{T}, \bar{x}^{I}, \bar{x}^{F}\right\rangle\right]$ $\cap \mathscr{N}_{(n)}^{<}$the set of all strictly increasing FNSVs in $\left[\left\langle\underline{x}^{T}, \underline{x}^{I}, \underline{x}^{F}\right\rangle\right.$, $\left.\left\langle\bar{x}^{T}, \bar{x}^{I}, \bar{x}^{F}\right\rangle\right]$. Then all the above general definitions concerning IFNSEvs and their types are modified by substituting $\mathbf{X}$ by $\mathbf{X}^{<}$.

Using Theorem 3.4, we describe necessary and sufficient conditions for six types of monotone IFNSEvs defined in Definition 3.1, which will be referred to as

$T 1, T 2, T 3, T 4, T 5, T 6$.

Theorem 3.5. $(T 1)$ Let IFNSM $\mathbf{A}=[\underline{A}, \bar{A}]$ and monotone IFNSV $\mathbf{X}^{<}$with strictly increasing bounds $\left\langle\underline{x}^{T}, \underline{x}^{I}, \underline{x}^{F}\right\rangle$,

$\left\langle\bar{x}^{T}, \bar{x}^{I}, \bar{x}^{F}\right\rangle$ be given. Then $\mathbf{X}^{<}$is a monotone strong FNSEv of $\mathbf{A}$ if and only if

$m^{*}(\bar{A}) \leq\left\langle\underline{x}^{T}, \underline{x}^{I}, \underline{x}^{F}\right\rangle,\left\langle\bar{x}^{T}, \bar{x}^{I}, \bar{x}^{F}\right\rangle \leq M^{*}(\underline{A})$.

Proof. Let $\mathbf{X}^{<}$is a monotone strong FNSEv of $\mathbf{A}$.

Then $A \otimes\left\langle x^{T}, x^{I}, x^{F}\right\rangle=\left\langle x^{T}, x^{I}, x^{F}\right\rangle$ holds for every $A \in \mathbf{A}$ and every strictly increasing FNSV $\left\langle x^{T}, x^{I}, x^{F}\right\rangle \in \mathbf{X}^{<}$. In particular,

$\bar{A} \otimes\left\langle\underline{T}^{T}, \underline{x}^{I}, \underline{x}^{F}\right\rangle=\left\langle\underline{x}^{T}, \underline{x}^{I}, \underline{x}^{F}\right\rangle, \underline{A} \otimes\left\langle\bar{x}^{T}, \bar{x}^{I}, \bar{x}^{F}\right\rangle=\left\langle\bar{x}^{T}, \bar{x}^{I}, \bar{x}^{F}\right\rangle$.

In view of Theorem 3.4 we immediately get the inequalities in (3.1).

To prove the converse implication, let us assume that the inequalities in (3.1) hold true. Then for every $A \in \mathbf{A}$ and every strictly increasing FNSV $\left\langle x^{T}, x^{I}, x^{F}\right\rangle \in \mathbf{X}^{<}$we get by the monotonicity results

$$
\begin{gathered}
m^{*}(A) \leq m^{*}(\bar{A}) \leq\left\langle\underline{x}^{T}, \underline{x}^{I}, \underline{x}^{F}\right\rangle \leq\left\langle x^{T}, x^{I}, x^{F}\right\rangle \leq\left\langle\bar{x}^{T}, \bar{x}^{I}, \bar{x}^{F}\right\rangle \leq \\
M^{*}(\underline{A}) \leq M^{*}(A) .
\end{gathered}
$$

Hence, $m^{*}(A) \leq\left\langle x^{T}, x^{I}, x^{F}\right\rangle \leq M^{*}(A)$ and $A \otimes\left\langle x^{T}, x^{I}, x^{F}\right\rangle=$ $\left\langle x^{T}, x^{I}, x^{F}\right\rangle$, in view of Theorem 3.4. In other words $\mathbf{X}^{<}$is a monotone strong FNSEv of $\mathbf{A}$.

Theorem 3.6. $(T 2)$ Let IFNSM A $=[\underline{A}, \bar{A}]$ and monotone IFNSV $\mathbf{X}^{<}=\left[\left\langle\underline{x}^{T}, \underline{x}^{I}, \underline{x}^{F}\right\rangle,\left\langle\bar{x}^{T}, \bar{x}^{I}, \bar{x}^{F}\right\rangle\right]$ with strictly increasing bounds $\left\langle\underline{x}^{T}, \underline{x}^{I}, \underline{x}^{F}\right\rangle,\left\langle\bar{x}^{T}, \bar{x}^{I}, \bar{x}^{F}\right\rangle$ be given. Then $\mathbf{X}^{<}$is a monotone strong universal FNSEv of $\mathbf{A}$ if and only if

$$
\begin{gathered}
m^{*}(\bar{A}) \leq\left\langle\bar{x}^{T}, \bar{x}^{I}, \bar{x}^{F}\right\rangle,\left\langle\underline{x}^{T}, \underline{x}^{I}, \underline{x}^{F}\right\rangle \leq \\
M^{*}(\underline{A}),\left\langle m^{*}(\bar{A}), M^{*}(\underline{A})\right\rangle \cap \mathscr{N}_{(n)}^{<} \neq \phi .
\end{gathered}
$$

Proof. Let us assume that $\mathbf{X}^{<}$is a monotone strong universal FNSEv of $\mathbf{A}$, i.e. there exists strictly increasing FNSV $\left\langle x^{T}, x^{I}, x^{F}\right\rangle \in \mathbf{X}^{<}$such that

$A \otimes\left\langle x^{T}, x^{I}, x^{F}\right\rangle=\left\langle x^{T}, x^{I}, x^{F}\right\rangle$ holds for every $A \in \mathbf{A}$, in particular,

$\underline{A} \otimes\left\langle x^{T}, x^{I}, x^{F}\right\rangle=\left\langle x^{T}, x^{I}, x^{F}\right\rangle$ and $\bar{A} \otimes\left\langle x^{T}, x^{I}, x^{F}\right\rangle=\left\langle x^{T}, x^{I}, x^{F}\right\rangle$. In view of Theorem 3.4, we get the inequalities $m^{*}(\bar{A}) \leq$ $\left\langle x^{T}, x^{I}, x^{F}\right\rangle \leq M^{*}(\underline{A})$, which directly imply all three conditions in (3.2).

To prove the converse implication, let us assume that the conditions in (3.2) hold true, i.e. $m^{*}(\bar{A}) \leq\left\langle\bar{x}^{T}\right.$,

$\left.\bar{x}^{I}, \bar{x}^{F}\right\rangle,\left\langle\underline{x}^{T}, \underline{x}^{I}, \underline{x}^{F}\right\rangle \leq M^{*}(\underline{A})$ and there is a strictly increasing FNSV $\left\langle x^{T}, x^{I}, x^{F}\right\rangle^{\prime}$ with $m^{*}(\bar{A}) \leq\left\langle x^{T}, x^{I}, x^{F}\right\rangle^{\prime} \leq M^{*}(\underline{A})$. Let us denote

$\left\langle x^{T}, x^{I}, x^{F}\right\rangle=\left(\left\langle x^{T}, x^{I}, x^{F}\right\rangle^{\prime} \vee\left\langle\underline{x}^{T}, \underline{x}^{I}, \underline{x}^{F}\right\rangle\right) \wedge\left\langle\bar{x}^{T}, \bar{x}^{I}, \bar{x}^{F}\right\rangle$. Using distributivity of operations $\wedge, \vee$, it is easy to show that $\left\langle x^{T}, x^{I}, x^{F}\right\rangle=\left(\left\langle x^{T}, x^{I}, x^{F}\right\rangle^{\prime} \wedge\left\langle\bar{x}^{T}, \bar{x}^{I}, \bar{x}^{F}\right\rangle\right) \vee\left\langle\underline{x}^{T}, \underline{x}^{I}, \underline{x}^{F}\right\rangle$ and it is also clear that $\left\langle\underline{x}^{T}, \underline{x}^{I}, \underline{x}^{F}\right\rangle \leq\left\langle x^{T}, x^{I}, x^{F}\right\rangle \leq\left\langle\bar{x}^{T}, \bar{x}^{I}, \bar{x}^{F}\right\rangle$. By assumption, FNSVs

$\left\langle x^{T}, x^{I}, x^{F}\right\rangle^{\prime},\left\langle\underline{x}^{T}, \underline{x}^{I}, \underline{x}^{F}\right\rangle,\left\langle\bar{x}^{T}, \bar{x}^{I}, \bar{x}^{F}\right\rangle$ are strictly increasing. As the operations $\wedge, \vee$ preserve strict monotonicity, FNSV $\left\langle x^{T}, x^{I}, x^{F}\right\rangle$ is strictly increasing, too. Further, we have

$$
\begin{gathered}
m^{*}(\bar{A}) \leq\left\langle x^{T}, x^{I}, x^{F}\right\rangle^{\prime} \wedge\left\langle\bar{x}^{T}, \bar{x}^{I}, \bar{x}^{F}\right\rangle \leq\left\langle x^{T}, x^{I}, x^{F}\right\rangle \leq \\
\left\langle x^{T}, x^{I}, x^{F}\right\rangle^{\prime} \vee\left\langle\underline{x}^{T}, \underline{x}^{I}, \underline{x}^{F}\right\rangle \leq M^{*}(\underline{A}) .
\end{gathered}
$$

As a consequence, for any FNSM $A \in \mathbf{A}$ we get

$$
m^{*}(A) \leq m^{*}(\bar{A}) \leq\left\langle x^{T}, x^{I}, x^{F}\right\rangle \leq M^{*}(\underline{A}) \leq M^{*}(A),
$$

i.e. $A \otimes\left\langle x^{T}, x^{I}, x^{F}\right\rangle=\left\langle x^{T}, x^{I}, x^{F}\right\rangle$. Thus, $\mathbf{X}^{<}$is a monotone strong universal FNSEv of $\mathbf{A}$.

Theorem 3.7. $(T 3)$ Let IFNSM $\mathbf{A}=[\underline{A}, \bar{A}]$ and monotone IFNSV

$\mathbf{X}^{<}=\left[\left\langle\underline{x}^{T}, \underline{x}^{I}, \underline{x}^{F}\right\rangle,\left\langle\bar{x}^{T}, \bar{x}^{I}, \bar{x}^{F}\right\rangle\right]$ with strictly increasing bounds $\left\langle\underline{x}^{T}, \underline{x}^{I}, \underline{x}^{F}\right\rangle,\left\langle\bar{x}^{T}, \bar{x}^{I}, \bar{x}^{F}\right\rangle$ be given. Then $\mathbf{X}^{<}$is a monotone universal

FNSEv of $\mathbf{A}$ if and only if

$$
\begin{aligned}
& m^{*}(\bar{A}) \leq\left\langle\bar{x}^{T}, \bar{x}^{I}, \bar{x}^{F}\right\rangle,\left\langle\underline{x}^{T}, \underline{x}^{I}, \underline{x}^{F}\right\rangle \\
& \leq M^{*}(\underline{A}),(\forall A \in \mathbf{A})\left[\left\langle m^{*}(\bar{A}), M^{*}(\underline{A})\right\rangle \cap \mathscr{N}_{(n)}^{<} \neq \boldsymbol{\phi}\right] .
\end{aligned}
$$

Proof. Let us assume that $\mathbf{X}^{<}$is a monotone universal FNSEv of $\mathbf{A}$, i.e. for every $A \in \mathbf{A}$ there exists strictly increasing FNSV $\left\langle x^{T}, x^{I}, x^{F}\right\rangle \in \mathbf{X}^{<}$such that

$A \otimes\left\langle x^{T}, x^{I}, x^{F}\right\rangle=\left\langle x^{T}, x^{I}, x^{F}\right\rangle$ holds. In particular, there exists $\left\langle x^{T}, x^{I}, x^{F}\right\rangle^{\prime},\left\langle x^{T}, x^{I}, x^{F}\right\rangle^{\prime \prime} \in \mathbf{X}^{<}$such that

$\underline{A} \otimes\left\langle x^{T}, x^{I}, x^{F}\right\rangle^{\prime}=\left\langle x^{T}, x^{I}, x^{F}\right\rangle^{\prime}$ and

$\bar{A} \otimes\left\langle x^{T}, x^{I}, x^{F}\right\rangle^{\prime \prime}=\left\langle x^{T}, x^{I}, x^{F}\right\rangle^{\prime \prime}$. In view of Theorem 3.4, we get the inequalities $\left\langle\underline{x}^{T}, \underline{x}^{I}, \underline{x}^{F}\right\rangle \leq\left\langle x^{T}, x^{I}, x^{F}\right\rangle^{\prime} \leq M^{*}(\underline{A})$,

$m^{*}(\bar{A}) \leq\left\langle x^{T}, x^{I}, x^{F}\right\rangle^{\prime \prime} \leq\left\langle\bar{x}^{T}, \bar{x}^{I}, \bar{x}^{F}\right\rangle$. The third condition in (3.3) follows directly from the assumption and from Theorem 3.4 .

To prove the converse implication, let us assume that the conditions in (3.3) hold true, i.e.

$m^{*}(\bar{A}) \leq\left\langle\bar{x}^{T}, \bar{x}^{I}, \bar{x}^{F}\right\rangle,\left\langle\underline{x}^{T}, \underline{x}^{I}, \underline{x}^{F}\right\rangle \leq M^{*}(\underline{A})$ and for every $A \in$ A, there is a strictly increasing FNSV $\left\langle x^{T}, x^{I}, x^{F}\right\rangle^{\prime}$ with $m^{*}(A) \leq$ $\left\langle x^{T}, x^{I}, x^{F}\right\rangle^{\prime} \leq M^{*}(A)$. Let FNSM $A$ and FNSV $\left\langle x^{T}, x^{I}, x^{F}\right\rangle^{\prime}$ 
be fixed and let us denote $\left\langle x^{T}, x^{I}, x^{F}\right\rangle=\left(\left\langle x^{T}, x^{I}, x^{F}\right\rangle^{\prime} \vee\left\langle\underline{x}^{T}, \underline{x}^{I}, \underline{x}^{F}\right\rangle\right) \wedge\left\langle\bar{x}^{T}, \bar{x}^{I}, \bar{x}^{F}\right\rangle$. Similarly as in the above proof, we show easily that $\left\langle x^{T}, x^{I}, x^{F}\right\rangle=$ $\left(\left\langle x^{T}, x^{I}, x^{F}\right\rangle^{\prime} \wedge\left\langle\bar{x}^{T}, \bar{x}^{I}, \bar{x}^{F}\right\rangle\right) \vee\left\langle\underline{x}^{T}, x^{I}, \underline{x}^{F}\right\rangle$ and $\left\langle x^{T}, x^{I}, x^{F}\right\rangle \in \mathbf{X}^{<}$. By the assumption we have $m^{*}(A) \leq m^{*}(\bar{A}) \leq\left\langle\bar{x}^{T}, \bar{x}^{I}, \bar{x}^{F}\right\rangle$ and $\left\langle\underline{x}^{T}, \underline{x}^{I}, \underline{x}^{F}\right\rangle \leq M^{*}(\underline{A}) \leq M^{*}(A)$, which implies

$$
\begin{gathered}
m^{*}(A) \leq\left\langle x^{T}, x^{I}, x^{F}\right\rangle^{\prime} \wedge\left\langle\bar{x}^{T}, \bar{x}^{I}, \bar{x}^{F}\right\rangle \leq\left\langle x^{T}, x^{I}, x^{F}\right\rangle \leq \\
\left\langle x^{T}, x^{I}, x^{F}\right\rangle^{\prime} \vee\left\langle\underline{x}^{T}, \underline{x}^{I}, \underline{x}^{F}\right\rangle \leq M^{*}(\underline{A}) .
\end{gathered}
$$

As a consequence, we get $m^{*}(A) \leq\left\langle x^{T}, x^{I}, x^{F}\right\rangle \leq M^{*}(A)$, i.e. $A \otimes\left\langle x^{T}, x^{I}, x^{F}\right\rangle=\left\langle x^{T}, x^{I}, x^{F}\right\rangle$. As the fixed FNSM $A \in \mathbf{A}$ is arbitrary, we have proved that $\mathbf{X}^{<}$is a monotone universal FNSEv of A.

Theorem 3.8. (T4) Let IFNSM A $=[\underline{A}, \bar{A}]$ and monotone IFNSV

$\mathbf{X}^{<}=\left[\left\langle\underline{x}^{T}, \underline{x}^{I}, \underline{x}^{F}\right\rangle,\left\langle\bar{x}^{T}, \bar{x}^{I}, \bar{x}^{F}\right\rangle\right]$ with strictly increasing bounds $\left\langle\underline{x}^{T}, \underline{x}^{I}, \underline{x}^{F}\right\rangle,\left\langle\bar{x}^{T}, \bar{x}^{I}, \bar{x}^{F}\right\rangle$ be given. Further let us denote

$$
\tilde{\mathbf{A}}=\left\{A \in \mathbf{A} ; m^{*}(A) \leq\left\langle\underline{x}^{T}, \underline{x}^{I}, \underline{x}^{F}\right\rangle\right\}, \tilde{A}=\bigvee \tilde{\mathbf{A}} .
$$

Then $\mathbf{X}^{<}$be monotone strong tolerance FNSEv of $\mathbf{A}$ if and only if

$m^{*}(\underline{A}) \leq\left\langle\underline{x}^{T}, \underline{x}^{I}, \underline{x}^{F}\right\rangle, \quad\left\langle\underline{x}^{T}, \underline{x}^{I}, \underline{x}^{F}\right\rangle \leq M^{*}(\tilde{A})$.

Proof. Let $\mathbf{X}^{<}$be monotone strong tolerance FNSEv of $\mathbf{A}$. Then there exists FNSM $A \in \mathbf{A}$ such that $A \otimes\left\langle x^{T}, x^{I}, x^{F}\right\rangle=$ $\left\langle x^{T}, x^{I}, x^{F}\right\rangle$ holds for every FNSV $\left\langle x^{T}, x^{I}, x^{F}\right\rangle \in \mathbf{X}^{<}$. In particular we have $A \otimes\left\langle\underline{x}^{T}, \underline{x}^{I}, \underline{x}^{F}\right\rangle=\left\langle\underline{x}^{T}, \underline{x}^{I}, \underline{x}^{F}\right\rangle, A \otimes\left\langle\bar{x}^{T}, \bar{x}^{I}, \bar{x}^{F}\right\rangle=$ $\left\langle\bar{x}^{T}, \bar{x}^{I}, \bar{x}^{F}\right\rangle$ and $m^{*}(A) \leq\left\langle\underline{x}^{T}, \underline{x}^{I}, \underline{x}^{F}\right\rangle \leq\left\langle\bar{x}^{T}, \bar{x}^{I}, \bar{x}^{F}\right\rangle \leq M^{*}(A)$. Hence $A \in \tilde{\mathbf{A}}$, which implies $\underline{A} \leq A \leq \tilde{A}, m^{*}(\underline{A}) \leq m^{*}(A) \leq$ $\left\langle\underline{x}^{T}, \underline{x}^{I}, \underline{x}^{F}\right\rangle$ and $\left\langle\bar{x}^{T}, \bar{x}^{I}, \bar{x}^{F}\right\rangle \leq \bar{M}^{*}(A) \leq M^{*}(\tilde{A})$.

Conversely, let $m^{*}(\underline{A}) \leq\left\langle\underline{x}^{T}, \underline{x}^{I}, \underline{x}^{F}\right\rangle($ i.e. $\underline{A} \in \tilde{\mathbf{A}})$, and $\left\langle\bar{x}^{T}, \bar{x}^{I}, \bar{x}^{F}\right\rangle \leq M^{*}(\tilde{\tilde{A}})$. It is easy to verify that for any two FNSMs $A^{\prime}, A^{\prime \prime} \in \tilde{\mathbf{A}}$ also their join $A^{\prime} \vee A^{\prime \prime}$ belongs to $\mathbf{A}$. In other words, $\tilde{\mathbf{A}}$ is closed under the operation $\vee$. As a consequence, $\tilde{A} \in \tilde{\mathbf{A}}$ and

$$
\begin{gathered}
m^{*}(\tilde{A}) \leq\left\langle\underline{x}^{T}, \underline{x}^{I}, \underline{x}^{F}\right\rangle \leq\left\langle x^{T}, x^{I}, x^{F}\right\rangle \leq\left\langle\bar{x}^{T}, \bar{x}^{I}, \bar{x}^{F}\right\rangle \leq M^{*}(\tilde{A}), \\
\tilde{A} \otimes\left\langle x^{T}, x^{I}, x^{F}\right\rangle=\left\langle x^{T}, x^{I}, x^{F}\right\rangle
\end{gathered}
$$

holds for every $\left\langle x^{T}, x^{I}, x^{F}\right\rangle \in \mathbf{X}^{<}$.

Hence, $\mathbf{X}^{<}$is monotone strong tolerance FNSEv of $\mathbf{A}$.

Theorem 3.9. $(T 5)$ Let IFNSM A $=[\underline{A}, \bar{A}]$ and monotone IFNSV

$\mathbf{X}^{<}=\left[\left\langle\underline{x}^{T}, \underline{x}^{I}, \underline{x}^{F}\right\rangle,\left\langle\bar{x}^{T}, \bar{x}^{I}, \bar{x}^{F}\right\rangle\right]$ with strictly increasing bounds $\left\langle\underline{x}^{T}, \underline{x}^{I}, \underline{x}^{F}\right\rangle,\left\langle\bar{x}^{T}, \bar{x}^{I}, \bar{x}^{F}\right\rangle$ be given. Then $\mathbf{X}^{<}$is a monotone tolerance FNSEv of A if and only if

$m^{*}(\underline{A}) \leq\left\langle\underline{x}^{T}, \underline{x}^{I}, \underline{x}^{F}\right\rangle,\left\langle\bar{x}^{T}, \bar{x}^{I}, \bar{x}^{F}\right\rangle \leq M^{*}(\bar{A})$.

Proof. Let $\mathbf{X}^{<}$be monotone tolerance FNSEv of $\mathbf{A}$. Then for every FNSV $\left\langle x^{T}, x^{I}, x^{F}\right\rangle \in \mathbf{X}^{<}$there exists FNSM $A \in \mathbf{A}$ such that $A \otimes\left\langle x^{T}, x^{I}, x^{F}\right\rangle=\left\langle x^{T}, x^{I}, x^{F}\right\rangle$. In particular, there are FNSMs $A^{\prime}, A^{\prime \prime} \in \mathbf{A}$ with $A^{\prime} \otimes\left\langle\underline{x}^{T}, \underline{x}^{I}, \underline{x}^{F}\right\rangle=\left\langle\underline{x}^{T}, \underline{x}^{I}, \underline{x}^{F}\right\rangle$, $A^{\prime \prime} \otimes\left\langle\bar{x}^{T}, \bar{x}^{I}, \bar{x}^{F}\right\rangle=\left\langle\bar{x}^{T}, \bar{x}^{I}, \bar{x}^{F}\right\rangle$, which gives $m^{*}(\underline{A}) \leq m^{*}\left(A^{\prime}\right) \leq$ $\left\langle x^{T}, x^{I}, x^{F}\right\rangle$ and $\left\langle\bar{x}^{T}, \bar{x}^{I}, \bar{x}^{F}\right\rangle \leq M^{*}\left(A^{\prime \prime}\right) \leq M^{*}(\bar{A})$.

Conversely, let conditions (3.5) be fulfilled and let $\left\langle x^{T}, x^{I}, x^{F}\right\rangle \in \mathbf{X}^{<}$be arbitrary, but fixed.

Define FNSM $A \in \mathscr{N}_{(n, n)}$ by putting, for every $j, k \in N$,

$$
\left\langle a_{j k}^{T}, a_{j k}^{I}, a_{j k}^{F}\right\rangle=\left\{\begin{array}{cc}
\left\langle\bar{a}_{j k}^{T}, \bar{a}_{j k}^{I}, \bar{a}_{j k}^{F}\right\rangle \wedge\left\langle x_{j}^{T}, x_{j}^{I}, x_{j}^{F}\right\rangle & j<k, \\
\left\langle\bar{a}_{j k}^{T}, \bar{a}_{j k}^{I}, \bar{a}_{j k}^{F}\right\rangle & j \leq k
\end{array}\right.
$$

For $i \in N$ we have, in view of the strict monotonicity of $\left\langle x^{T}, x^{I}, x^{F}\right\rangle$,

$$
\begin{aligned}
m_{i}^{*}(A) & =\max _{j \leq i} \max _{k>j}\left(\left\langle\bar{a}_{j k}^{T}, \bar{a}_{j k}^{I}, \bar{a}_{j k}^{F}\right\rangle \wedge\left\langle x_{j}^{T}, x_{j}^{I}, x_{j}^{F}\right\rangle\right) \\
& \leq \max _{j \leq i} \max _{k>j}\left(\left\langle\bar{a}_{j k}^{T}, \bar{a}_{j k}^{I}, \bar{a}_{j k}^{F}\right\rangle \wedge\left\langle x_{i}^{T}, x_{i}^{I}, x_{i}^{F}\right\rangle\right) \\
& =\left(\max _{j \leq i} \max _{k>j}\left\langle\bar{a}_{j k}^{T}, \bar{a}_{j k}^{I}, \bar{a}_{j k}^{F}\right\rangle\right) \wedge\left\langle x_{i}^{T}, x_{i}^{I}, x_{i}^{F}\right\rangle \\
& =m_{i}^{*}(\bar{A}) \wedge\left\langle x_{i}^{T}, x_{i}^{I}, x_{i}^{F}\right\rangle .
\end{aligned}
$$

Hence $m^{*}(A) \leq m^{*}(\bar{A}) \wedge\left\langle x^{T}, x^{I}, x^{F}\right\rangle \leq\left\langle x^{T}, x^{I}, x^{F}\right\rangle$. Further we have

$$
\begin{aligned}
M_{i}^{*}(A) & =\min _{j \geq i} \max _{k \geq j}\left\langle a_{j k}^{T}, a_{j k}^{I}, a_{j k}^{F}\right\rangle \\
& =\min _{j \geq i}\left(\left\langle\bar{a}_{k k}^{T}, \bar{a}_{k k}^{I}, \bar{a}_{k k}^{F}\right\rangle \vee \max _{k>j}\left(\left\langle\bar{a}_{j k}^{T}, \bar{a}_{j k}^{I}, \bar{a}_{j k}^{F}\right\rangle \wedge\left\langle x_{j}^{T}, x_{j}^{I}, x_{j}^{F}\right\rangle\right)\right) \\
& \geq \min _{j \geq i}\left(\left\langle\bar{a}_{k k}^{T}, \bar{a}_{k k}^{I}, \bar{a}_{k k}^{F}\right\rangle \vee \max _{k>j}\left(\left\langle\bar{a}_{j k}^{T}, \bar{a}_{j k}^{I}, \bar{a}_{j k}^{F}\right\rangle \wedge\left\langle x_{i}^{T}, x_{i}^{I}, x_{i}^{F}\right\rangle\right)\right) \\
& \geq \min _{j \geq i} \max _{k \geq j}\left(\left\langle\bar{a}_{j k}^{T}, \bar{a}_{j k}^{I}, \bar{a}_{j k}^{F}\right\rangle \wedge\left\langle x_{i}^{T}, x_{i}^{I}, x_{i}^{F}\right\rangle\right) \\
& =\left(\min _{j \geq i} \max _{k \geq j}\left\langle\bar{a}_{j k}^{T}, \bar{a}_{j k}^{I}, \bar{a}_{j k}^{F}\right\rangle\right) \wedge\left\langle x_{i}^{T}, x_{i}^{I}, x_{i}^{F}\right\rangle \\
& =M^{*}(\bar{A}) \wedge\left\langle x_{i}^{T}, x_{i}^{I}, x_{i}^{F}\right\rangle .
\end{aligned}
$$

Hence $M^{*}(A) \geq M^{*}(\bar{A}) \wedge\left\langle x^{T}, x^{I}, x^{F}\right\rangle \geq\left\langle\bar{x}^{T}, \bar{x}^{I}, \bar{x}^{F}\right\rangle \wedge\left\langle x^{T}, x^{I}, x^{F}\right\rangle$ $=\left\langle x^{T}, x^{I}, x^{F}\right\rangle$ and the inequalities $m^{*}(A) \leq\left\langle x^{T}, x^{I}, x^{F}\right\rangle \leq M^{*}(A)$ imply $A \otimes\left\langle x^{T}, x^{I}, x^{F}\right\rangle=\left\langle x^{T}, x^{I}, x^{F}\right\rangle$, in view of Theorem 3.4. We have shown that $\mathbf{X}^{<}$is a monotone tolerance FNSEv of $\mathbf{A}$.

Theorem 3.10. (T6) Let IFNSM A $=[\underline{A}, \bar{A}]$ and monotone IFNSV

$\mathbf{X}^{<}=\left[\left\langle\underline{x}^{T}, \underline{x}^{I}, \underline{x}^{F}\right\rangle,\left\langle\bar{x}^{T}, \bar{x}^{I}, \bar{x}^{F}\right\rangle\right]$ with strictly increasing bounds $\left\langle\underline{x}^{T}, \underline{x}^{I}, \underline{x}^{F}\right\rangle,\left\langle\bar{x}^{T}, \bar{x}^{I}, \bar{x}^{F}\right\rangle$ be given. Then $\mathbf{X}^{<}$is a monotone weak FNSEv of $\mathbf{A}$ if and only if

$$
\begin{aligned}
&\left\langle\underline{x}^{T}, \underline{x}^{I}, \underline{x}^{F}\right\rangle \leq M^{*}(\bar{A}), m^{*}(\underline{A}) \leq \\
&\left\langle\bar{x}^{T}, \bar{x}^{I}, \bar{x}^{F}\right\rangle, \quad\left\langle m^{*}(\underline{A}), M^{*}(\bar{A})\right\rangle \cap \mathscr{N}_{(n)}^{<} \neq \phi .
\end{aligned}
$$

Proof. Let us assume that $\mathbf{X}^{<}$is a monotone weak FNSEv of $\mathbf{A}$, i.e. there exists a FNSM $A \in \mathbf{A}$ and a strictly increasing FNSV $\left\langle x^{T}, x^{I}, x^{F}\right\rangle \in \mathbf{X}^{<}$such that $A \otimes\left\langle x^{T}, x^{I}, x^{F}\right\rangle=$ $\left\langle x^{T}, x^{I}, x^{F}\right\rangle$ holds. In view of Theorem 3.4, we have $m^{*}(\underline{A}) \leq$ $m^{*}(A) \leq\left\langle x^{T}, x^{I}, x^{F}\right\rangle \leq M^{*}(A) \leq M^{*}(\bar{A})$,

hence $\left\langle m^{*}(\underline{A}), M^{*}(\bar{A})\right\rangle \cap \mathscr{N}_{(n)}^{<} \neq \phi$. Moreover, the above inequalities imply 


$$
\begin{aligned}
& \left\langle\underline{x}^{T}, \underline{x}^{I}, \underline{x}^{F}\right\rangle \leq\left\langle x^{T}, x^{I}, x^{F}\right\rangle \leq M^{*}(A) \leq M^{*}(\bar{A}), \\
& m^{*}(\underline{A}) \leq m^{*}(A) \leq\left\langle x^{T}, x^{I}, x^{F}\right\rangle \leq\left\langle\bar{x}^{T}, \bar{x}^{I}, \bar{x}^{F}\right\rangle .
\end{aligned}
$$

To prove the converse implication, let us assume that the conditions in (3.6) hold true, i.e. $\left\langle\underline{x}^{T}, \underline{x}^{I}, \underline{x}^{F}\right\rangle \leq M^{*}(\bar{A}), m^{*}(\underline{A}) \leq$ $\left\langle\bar{x}^{T}, \bar{x}^{I}, \bar{x}^{F}\right\rangle$ and there is a strictly increasing FNSV $\left\langle x^{T}, x^{I}, x^{F}\right\rangle^{\prime}$ with $m^{*}(\underline{A}) \leq\left\langle x^{T}, x^{I}, x^{F}\right\rangle^{\prime} \leq M^{*}(\bar{A})$. We denote $\left\langle x^{T}, x^{I}, x^{F}\right\rangle=$ $\left(\left\langle x^{T}, x^{I}, x^{F}\right\rangle^{\prime} \vee\left\langle\underline{x}^{T}, \underline{x}^{I}, \underline{x}^{F}\right\rangle\right) \wedge\left\langle\bar{x}^{T}, \bar{x}^{I}, \bar{x}^{F}\right\rangle$. Similarly as in the proof of Theorem 3.6, it can be easily shown that $\left\langle x^{T}, x^{I}, x^{F}\right\rangle=$ $\left(\left\langle x^{T}, x^{I}, x^{F}\right\rangle^{\prime} \wedge\left\langle\bar{x}^{T}, \bar{x}^{I}, \bar{x}^{F}\right\rangle\right) \vee\left\langle\underline{x}^{T}, \underline{x}^{I}, \underline{x}^{F}\right\rangle$ and $\left\langle x^{T}, x^{I}, x^{F}\right\rangle \in \mathbf{X}^{<}$. Moreover, the inequalities $m^{*}(\underline{A}) \leq\left\langle x^{T}, x^{I}, x^{F}\right\rangle^{\prime} \leq M^{*}(\bar{A})$ together with assumption $\left\langle\underline{x}^{T}, \underline{x}^{I}, \underline{x}^{F}\right\rangle \leq M^{*}(\overline{\bar{A}})$,

$m^{*}(\underline{A}) \leq\left\langle\bar{x}^{T}, \bar{x}^{I}, \bar{x}^{F}\right\rangle$ imply

$$
\begin{gathered}
m^{*}(\underline{A}) \leq\left(\left\langle x^{T}, x^{I}, x^{F}\right\rangle^{\prime} \wedge\left\langle\bar{x}^{T}, \bar{x}^{I}, \bar{x}^{F}\right\rangle\right) \leq\left(\left\langle x^{T}, x^{I}, x^{F}\right\rangle^{\prime}\right. \\
\left.\wedge\left\langle\bar{x}^{T}, \bar{x}^{I}, \bar{x}^{F}\right\rangle\right) \vee\left\langle\underline{x}^{T}, \underline{x}^{I}, \underline{x}^{F}\right\rangle=\left\langle x^{T}, x^{I}, x^{F}\right\rangle=\left(\left\langle x^{T}, x^{I}, x^{F}\right\rangle^{\prime} \vee\right. \\
\left.\left\langle\underline{x}^{T}, \underline{x}^{I}, \underline{x}^{F}\right\rangle\right) \wedge\left\langle\bar{x}^{T}, \bar{x}^{I}, \bar{x}^{F}\right\rangle \leq\left\langle x^{T}, x^{I}, x^{F}\right\rangle^{\prime} \vee\left\langle\underline{x}^{T}, \underline{x}^{I}, \underline{x}^{F}\right\rangle \leq \\
M^{*}(\bar{A}) .
\end{gathered}
$$

Hence, $m^{*}(\underline{A}) \leq\left\langle x^{T}, x^{I}, x^{F}\right\rangle \leq M^{*}(\bar{A})$. Denoting

$$
\begin{gathered}
\left\langle\underline{x}^{T}, \underline{x}^{I}, \underline{x}^{F}\right\rangle^{\prime}=\bigwedge\left\{\left\langle y^{T}, y^{I}, y^{F}\right\rangle \in \mathbf{X}^{<} ; m^{*}(\underline{A}) \leq\right. \\
\left.\left\langle y^{T}, y^{I}, y^{F}\right\rangle\right\}\left\langle\bar{x}^{T}, \bar{x}^{I}, \bar{x}^{F}\right\rangle^{\prime}=\bigvee\left\{\left\langle y^{T}, y^{I}, y^{F}\right\rangle \in\right. \\
\left.\mathbf{X}^{<} ;\left\langle y^{T}, y^{I}, y^{F}\right\rangle \leq M^{*}(\bar{A})\right\},
\end{gathered}
$$

we get $\left\langle\underline{x}^{T}, \underline{x}^{I}, \underline{x}^{F}\right\rangle^{\prime} \leq\left\langle x^{T}, x^{I}, x^{F}\right\rangle \leq\left\langle\bar{x}^{T}, \bar{x}^{I}, \bar{x}^{F}\right\rangle^{\prime}$.

Clearly, the inequalities $m^{*}(\underline{A}) \leq\left\langle\underline{x}^{T}, \underline{x}^{I}, \underline{x}^{F}\right\rangle^{\prime}$ and $\left\langle\bar{x}^{T}, \bar{x}^{I}, \bar{x}^{F}\right\rangle^{\prime}$ $\leq M^{*}(\bar{A})$ holds true, which implies that the IFNSV $\mathbf{X}^{\prime}=$ $\left[\left\langle\underline{x}^{T}, \underline{x}^{I}, \underline{x}^{F}\right\rangle^{\prime},\left\langle\bar{x}^{T}, \bar{x}^{I}, \bar{x}^{F}\right\rangle^{\prime}\right]$ is monotone tolerance FNSEv of $\mathbf{A}$, in view of Theorem 3.9. As we have shown above, $\left\langle x^{T}, x^{I}, x^{F}\right\rangle$ belongs to $\mathbf{X}^{\prime}$, hence there exists $A \in \mathbf{A}$ with $m^{*}(A) \leq\left\langle x^{T}, x^{I}, x^{F}\right\rangle \leq M^{*}(A)$, i.e. $A \otimes\left\langle x^{T}, x^{I}, x^{F}\right\rangle=\left\langle x^{T}, x^{I}, x^{F}\right\rangle$.

\section{Relations between various types of monotone FNSEvs}

Theorem 4.1. Let IFNSM $\mathbf{A}=[\underline{A}, \bar{A}]$ and monotone IFNSV $\mathbf{X}^{<}=\left[\left\langle\underline{x}^{T}, \underline{x}^{I}, \underline{x}^{F}\right\rangle,\left\langle\bar{x}^{T}, \bar{x}^{I}, \bar{x}^{F}\right\rangle\right]$ with strictly increasing bounds $\left\langle\underline{x}^{T}, \underline{x}^{I}, \underline{x}^{F}\right\rangle,\left\langle\bar{x}^{T}, \bar{x}^{I}, \bar{x}^{F}\right\rangle$ be given. Then the following implications hold true.

$(T 1) \Rightarrow(T 2)$ If $\mathbf{X}^{<}$is a monotone strong FNSEv of $\mathbf{A}$, then $\mathbf{X}^{<}$is a monotone strong universal FNSEv of A,

$(T 1) \Rightarrow(T 4)$ If $\mathbf{X}^{<}$is a monotone strong FNSEv of $\mathbf{A}$, then $\mathbf{X}^{<}$is a monotone strong tolerance FNSEv of $\mathbf{A}$,

$(T 3) \Rightarrow(T 6)$ If $\mathbf{X}^{<}$is a monotone universal FNSEv of $\mathbf{A}$, then $\mathbf{X}^{<}$is a monotone weak FNSEv of $\mathbf{A}$,

$(T 5) \Rightarrow(T 6)$ if $\mathbf{X}^{<}$is a monotone tolerance FNSEv of $\mathbf{A}$, then $\mathbf{X}^{<}$is a monotone weak universal FNSEv of $\mathbf{A}$,

Proof. The implications follow directly from Definition 3.1.

Remark 4.2. It is easy to shown that converse implications to those in Theorem 4.1 do not hold true.

Theorem 4.3. $(T 2 \Rightarrow T 3)$ Let IFNSM $A=[\underline{A}, \bar{A}]$ and monotone IFNSV $\mathbf{X}^{<}=\left[\left\langle\underline{x}^{T}, \underline{x}^{I}, \underline{x}^{F}\right\rangle,\left\langle\bar{x}^{T}, \bar{x}^{I}, \bar{x}^{F}\right\rangle\right]$ with strictly increasing bounds $\left\langle\underline{x}^{T}, \underline{x}^{I}, \underline{x}^{F}\right\rangle,\left\langle\bar{x}^{T}, \bar{x}^{I}, \bar{x}^{F}\right\rangle$ be given. If $\mathbf{X}^{<}$is a monotone strong universal FNSEv of $\mathbf{A}$, then $\mathbf{X}^{<}$is a monotone universal FNSEv of A.

Proof. The implication follows directly from Definition 3.1.

The converse implication is not true as the next example shows.

Example 4.4. $(T 3 \nRightarrow T 2)$ Let $\bar{A}, \underline{A}$ and $\left\langle\underline{x}^{T}, \underline{x}^{I}, \underline{x}^{F}\right\rangle,\left\langle\bar{x}^{T}, \bar{x}^{I}, \bar{x}^{F}\right\rangle$ have the form

$$
\begin{gathered}
\underline{A}=\left[\begin{array}{ll}
\langle 0.2,0.1,0.8\rangle & \langle 0.2,0.1,0.8\rangle \\
\langle 0.3,0.2,0.7\rangle & \langle 0.4,0.3,0.6\rangle,
\end{array}\right], \\
\bar{A}=\left[\begin{array}{ll}
\langle 0.2,0.1,0.8\rangle & \langle 0.3,0.2,0.7\rangle \\
\langle 0.3,0.2,0.7\rangle & \langle 0.5,0.4,0.6\rangle
\end{array}\right] \\
\left\langle\underline{x}^{T}, \underline{x}^{I}, \underline{x}^{F}\right\rangle=\left[\begin{array}{l}
\langle 0.2,0.1,0.8\rangle \\
\langle 0.3,0.2,0.7\rangle
\end{array}\right], \\
\left\langle\bar{x}^{T}, \bar{x}^{I}, \bar{x}^{F}\right\rangle=\left[\begin{array}{l}
\langle 0.3,0.2,0.7\rangle \\
\langle 0.4,0.3,0.6\rangle
\end{array}\right] .
\end{gathered}
$$

Then for every FNSM $A \in \mathbf{A}$ of the form

$$
\begin{gathered}
A=\left[\begin{array}{ll}
\langle 0.2,0.1,0.8\rangle & \left\langle k^{T}, k^{I}, k^{F}\right\rangle \\
\langle 0.3,0.2,0.7\rangle & \left\langle s^{T}, s^{I}, s^{F}\right\rangle
\end{array}\right], \\
\langle 0.2,0.1,0.8\rangle \leq\left\langle k^{T}, k^{I}, k^{F}\right\rangle \leq\langle 0.3,0.2,0.7\rangle, \\
\langle 0.4,0.3,0.6\rangle \leq\left\langle s^{T}, s^{I}, s^{F}\right\rangle \leq\langle 0.5,0.4,0.6\rangle, \\
\text { strictly increasing FNSV } \\
{\left[\begin{array}{c}
\left\langle k^{T}, k^{I}, k^{F}\right\rangle \\
\langle 0.4,0.3,0.6\rangle
\end{array}\right] \text {. belongs to }} \\
\left\langle\left[\begin{array}{l}
\left\langle k^{T}, k^{I}, k^{F}\right\rangle \\
\left\langle k^{T}, k^{I}, k^{F}\right\rangle
\end{array}\right],\left[\begin{array}{l}
\left\langle k^{T}, k^{I}, k^{F}\right\rangle \\
\left\langle s^{T}, s^{I}, s^{F}\right\rangle
\end{array}\right]\right\rangle=\left\langle m^{*}(A), M^{*}(A)\right\rangle,
\end{gathered}
$$

that means $\mathbf{X}^{<}$is a monotone universal FNSEv of $\mathbf{A}$ but $\mathbf{X}^{<}$is not a monotone strong universal FNSEv of $\mathbf{A}$ because

$$
\begin{aligned}
& \left\langle m^{*}(\bar{A}), M^{*}(\underline{A})\right\rangle=\left\langle\left[\begin{array}{c}
\langle 0.3,0.2,0.7\rangle \\
\langle 0.3,0.2,0.7\rangle
\end{array}\right],\left[\begin{array}{l}
\langle 0.2,0.1,0.8\rangle \\
\langle 0.4,0.3,0.6\rangle
\end{array}\right]\right\rangle \cap \\
& \mathscr{N}_{(n)}^{<}=\phi .
\end{aligned}
$$

Remark 4.5. Monotone interval FNSV $\mathbf{X}^{<}$in the previous example fails to be a monotone strong universal FNSEv of $\mathbf{A}$ because the third condition in (3.2) is not fulfilled. We may note that in the example the first two conditions in (3.2) are satisfied, hence these two conditions together are not sufficient for the monotone strong universality of $\mathbf{X}^{<}$.

Theorem 4.6. $(T 4 \Rightarrow T 5)$ Let interval FNSM $A=[\underline{A}, \bar{A}]$ and monotone IFNSV $\mathbf{X}^{<}=\left[\left\langle\underline{x}^{T}, \underline{x}^{I}, \underline{x}^{F}\right\rangle,\left\langle\bar{x}^{T}, \bar{x}^{I}, \bar{x}^{F}\right\rangle\right]$ with strictly increasing bounds $\left\langle\underline{x}^{T}, \underline{x}^{I}, \underline{x}^{F}\right\rangle,\left\langle\bar{x}^{T}, \bar{x}^{I}, \bar{x}^{F}\right\rangle$ be given. If $\mathbf{X}^{<}$is a monotone strong tolerance FNSEv of $\mathbf{A}$, then $\mathbf{X}^{<}$is a monotone tolerance FNSEv of A.

Proof. The implication follows directly from Definition 3.1.

The next example shows that the converse implication is not true. 
Example 4.7. $(T 5 \nRightarrow T 4)$ Let $\underline{A}, \bar{A}$ and $\left\langle\underline{x}^{T}, \underline{x}^{I}, \underline{x}^{F}\right\rangle,\left\langle\bar{x}^{T}, \bar{x}^{I}, \bar{x}^{F}\right\rangle$ have the form

$$
\begin{gathered}
\underline{A}=\left[\begin{array}{ll}
\langle 0.2,0.1,0.8\rangle & \langle 0.3,0.2,0.7\rangle \\
\langle 0.3,0.2,0.7\rangle & \langle 0.4,0.3,0.6\rangle,
\end{array}\right], \\
\bar{A}=\left[\begin{array}{ll}
\langle 0.2,0.1,0.8\rangle & \langle 0.4,0.3,0.6\rangle \\
\langle 0.3,0.2,0.7\rangle & \langle 0.4,0.3,0.6\rangle
\end{array}\right] \\
\left\langle\underline{x}^{T}, \underline{x}^{I}, \underline{x}^{F}\right\rangle=\left[\begin{array}{l}
\langle 0.3,0.2,0.7\rangle \\
\langle 0.4,0.3,0.6\rangle
\end{array}\right], \\
\left\langle\bar{x}^{T}, \bar{x}^{I}, \bar{x}^{F}\right\rangle=\left[\begin{array}{l}
\langle 0.4,0.3,0.6\rangle \\
\langle 0.4,0.3,0.6\rangle
\end{array}\right] . \\
\text { with } m^{*}(\underline{A})=\left[\begin{array}{l}
\langle 0.3,0.2,0.7\rangle \\
\langle 0.3,0.2,0.7\rangle
\end{array}\right], M^{*}(\bar{A})=\left[\begin{array}{l}
\langle 0.4,0.3,0.6\rangle \\
\langle 0.4,0.3,0.6\rangle
\end{array}\right] .
\end{gathered}
$$

The following inequalities

$$
\begin{gathered}
m^{*}(\underline{A})=\left[\begin{array}{l}
\langle 0.3,0.2,0.7\rangle \\
\langle 0.3,0.2,0.7\rangle
\end{array}\right] \leq\left\langle\underline{x}^{T}, \underline{x}^{I}, \underline{x}^{F}\right\rangle=\left[\begin{array}{l}
\langle 0.3,0.2,0.7\rangle \\
\langle 0.4,0.3,0.6\rangle
\end{array}\right], \\
\left\langle\bar{x}^{T}, \bar{x}^{I}, \bar{x}^{F}\right\rangle=\left[\begin{array}{l}
\langle 0.4,0.3,0.6\rangle \\
\langle 0.4,0.3,0.6\rangle
\end{array}\right] \leq M^{*}(\bar{A})=\left[\begin{array}{l}
\langle 0.4,0.3,0.6\rangle \\
\langle 0.4,0.3,0.6\rangle
\end{array}\right]
\end{gathered}
$$

that means $\mathbf{X}^{<}$is a monotone tolerance FNSEv of $\mathbf{A}$ but $\mathbf{X}^{<}$is not a monotone strong tolerance FNSEv of $\mathbf{A}$ because

$$
\begin{gathered}
\tilde{A}=\left[\begin{array}{cc}
\langle 0.2,0.1,0.8\rangle & \langle 0.3,0.2,0.7\rangle \\
\langle 0.3,0.2,0.7\rangle & \langle 0.4,0.3,0.6\rangle,
\end{array}\right], \text { and } \\
\left\langle\bar{x}^{T}, \bar{x}^{I}, \bar{x}^{F}\right\rangle=\left[\begin{array}{l}
\langle 0.4,0.3,0.6\rangle \\
\langle 0.4,0.3,0.6\rangle
\end{array}\right] \not \leq M^{*}(\tilde{A})=\left[\begin{array}{l}
\langle 0.3,0.2,0.7\rangle \\
\langle 0.4,0.3,0.6\rangle
\end{array}\right] .
\end{gathered}
$$

Next two examples indicate further non-implication between types of monotone IFNSEvs.

Example 4.8. $(T 4 \nRightarrow T 3)$ Let $\underline{A}, \bar{A}$ and $\left\langle\underline{x}^{T}, \underline{x}^{I}, \underline{x}^{F}\right\rangle,\left\langle\bar{x}^{T}, \bar{x}^{I}, \bar{x}^{F}\right\rangle$ have the form

$$
\begin{gathered}
\underline{A}=\left[\begin{array}{ll}
\langle 0.3,0.2,0.7\rangle & \langle 0.2,0.1,0.8\rangle \\
\langle 0.3,0.2,0.7\rangle & \langle 0.3,0.2,0.7\rangle
\end{array}\right], \\
\bar{A}=\left[\begin{array}{ll}
\langle 0.3,0.2,0.7\rangle & \langle 0.3,0.2,0.7\rangle \\
\langle 0.3,0.2,0.7\rangle & \langle 0.5,0.4,0.6\rangle
\end{array}\right], \\
\left\langle\underline{x}^{T}, \underline{x}^{I}, \underline{x}^{F}\right\rangle=\left[\begin{array}{l}
\langle 0.3,0.2,0.7\rangle \\
\langle 0.4,0.3,0.6\rangle
\end{array}\right], \\
\left\langle\bar{x}^{T}, \bar{x}^{I}, \bar{x}^{F}\right\rangle=\left[\begin{array}{l}
\langle 0.3,0.2,0.7\rangle \\
\langle 0.5,0.4,0.6\rangle
\end{array}\right] .
\end{gathered}
$$

Then we have

$$
\begin{aligned}
& m^{*}(\underline{A})=\left[\begin{array}{l}
\langle 0.2,0.1,0.8\rangle \\
\langle 0.2,0.1,0.8\rangle
\end{array}\right] \leq\left\langle\underline{x}^{T}, \underline{x}^{I}, \underline{x}^{F}\right\rangle \\
& \tilde{A}=\left[\begin{array}{ll}
\langle 0.3,0.2,0.7\rangle & \langle 0.3,0.2,0.7\rangle \\
\langle 0.3,0.2,0.7\rangle & \langle 0.5,0.4,0.6\rangle
\end{array}\right] \\
& \left\langle\bar{x}^{T}, \bar{x}^{I}, \bar{x}^{F}\right\rangle=\left[\begin{array}{l}
\langle 0.3,0.2,0.7\rangle \\
\langle 0.5,0.4,0.6\rangle
\end{array}\right] \leq M^{*}(\tilde{A})=\left[\begin{array}{l}
\langle 0.3,0.2,0.7\rangle \\
\langle 0.5,0.4,0.6\rangle
\end{array}\right]
\end{aligned}
$$

which means that $\mathbf{X}^{<}$is a monotone strong tolerance FNSEv of $\mathbf{A}$, but $\mathbf{X}^{<}$is not a monotone universal FNSEv of $\mathbf{A}$ because

$$
\left\langle\underline{x}^{T}, \underline{x}^{I}, \underline{x}^{F}\right\rangle=\left[\begin{array}{l}
\langle 0.3,0.2,0.7\rangle \\
\langle 0.4,0.3,0.6\rangle
\end{array}\right] \not \leq M^{*}(\underline{A})=\left[\begin{array}{l}
\langle 0.3,0.2,0.7\rangle \\
\langle 0.3,0.2,0.7\rangle
\end{array}\right] .
$$

Example 4.9. $(T 2 \nRightarrow T 5)$ Let $\underline{A}, \bar{A}$ and $\left\langle\underline{x}^{T}, \underline{x}^{I}, \underline{x}^{F}\right\rangle,\left\langle\bar{x}^{T}, \bar{x}^{I}, \bar{x}^{F}\right\rangle$ have the form

$$
\begin{gathered}
\underline{A}=\left[\begin{array}{ll}
\langle 0.2,0.1,0.8\rangle & \langle 0.3,0.2,0.7\rangle \\
\langle 0.3,0.2,0.7\rangle & \langle 0.4,0.3,0.6\rangle
\end{array}\right], \\
\bar{A}=\left[\begin{array}{ll}
\langle 0.2,0.1,0.8\rangle & \langle 0.3,0.2,0.7\rangle \\
\langle 0.3,0.2,0.7\rangle & \langle 0.5,0.4,0.6\rangle
\end{array}\right] \\
\left\langle\underline{x}^{T}, \underline{x}^{I}, \underline{x}^{F}\right\rangle=\left[\begin{array}{l}
\langle 0.2,0.1,0.8\rangle \\
\langle 0.4,0.3,0.6\rangle
\end{array}\right], \\
\left\langle\bar{x}^{T}, \bar{x}^{I}, \bar{x}^{F}\right\rangle=\left[\begin{array}{l}
\langle 0.5,0.4,0.6\rangle \\
\langle 0.6,0.5,0.3\rangle
\end{array}\right] .
\end{gathered}
$$

The following inequalities

$$
\begin{aligned}
& m^{*}(\bar{A})=\left[\begin{array}{l}
\langle 0.3,0.2,0.7\rangle \\
\langle 0.3,0.2,0.7\rangle
\end{array}\right] \leq\left\langle\bar{x}^{T}, \bar{x}^{I}, \bar{x}^{F}\right\rangle=\left[\begin{array}{l}
\langle 0.5,0.4,0.6\rangle \\
\langle 0.6,0.5,0.3\rangle
\end{array}\right] . \\
& \left\langle\underline{x}^{T}, \underline{x}^{I}, \underline{x}^{F}\right\rangle=\left[\begin{array}{l}
\langle 0.2,0.1,0.8\rangle \\
\langle 0.4,0.3,0.6\rangle
\end{array}\right] \leq M^{*}(\underline{A})=\left[\begin{array}{l}
\langle 0.3,0.2,0.7\rangle \\
\langle 0.4,0.3,0.6\rangle
\end{array}\right],
\end{aligned}
$$

$$
\begin{gathered}
\left\langle m^{*}(\bar{A}), M^{*}(\underline{A})\right\rangle \cap \mathscr{N}_{(n)}^{<}= \\
\left\langle\left[\begin{array}{l}
\langle 0.3,0.2,0.7\rangle \\
\langle 0.3,0.2,0.7\rangle
\end{array}\right],\left[\begin{array}{l}
\langle 0.3,0.2,0.7\rangle \\
\langle 0.4,0.3,0.6\rangle
\end{array}\right]\right\rangle \cap \mathscr{N}_{(n)}^{<} \neq \phi,
\end{gathered}
$$

that mean $\mathbf{X}^{<}$is a monotone strong universal FNSEv of $\mathbf{A}$, but $\mathbf{X}^{<}$is not a monotone tolerance FNSEv of $\mathbf{A}$ because

$$
\left.m^{*}(\underline{A})=\left[\begin{array}{l}
\langle 0.3,0.2,0.7\rangle \\
\langle 0.3,0.2,0.7\rangle
\end{array}\right] \not \underline{\underline{x}} \underline{x}^{T}, \underline{x}^{I}, \underline{x}^{F}\right\rangle=\left[\begin{array}{l}
\langle 0.2,0.1,0.8\rangle \\
\langle 0.4,0.3,0.6\rangle
\end{array}\right] .
$$

Remark 4.10. The previous two examples shown that implications $T 4 \Rightarrow T 3$ and $T 2 \Rightarrow T 5$ are not fulfilled. It can be easily seen that if $T 4 \nRightarrow T 3$ and $T 2 \nRightarrow T 5$, then also $T 2 \nRightarrow$ $T 4, T 3 \nRightarrow T 4, T 3 \nRightarrow T 5, T 4 \nRightarrow T 2, T 5 \nRightarrow T 2, T 5 \nRightarrow T 3$.

E.g. if the implication $T 2 \Rightarrow T 4$ holds true, then by $T 4 \Rightarrow T 5$ (Theorem 4.6) we get $T 2 \Rightarrow T 5$, a contradiction. The proofs of the remaining five non-implications are analogous. 


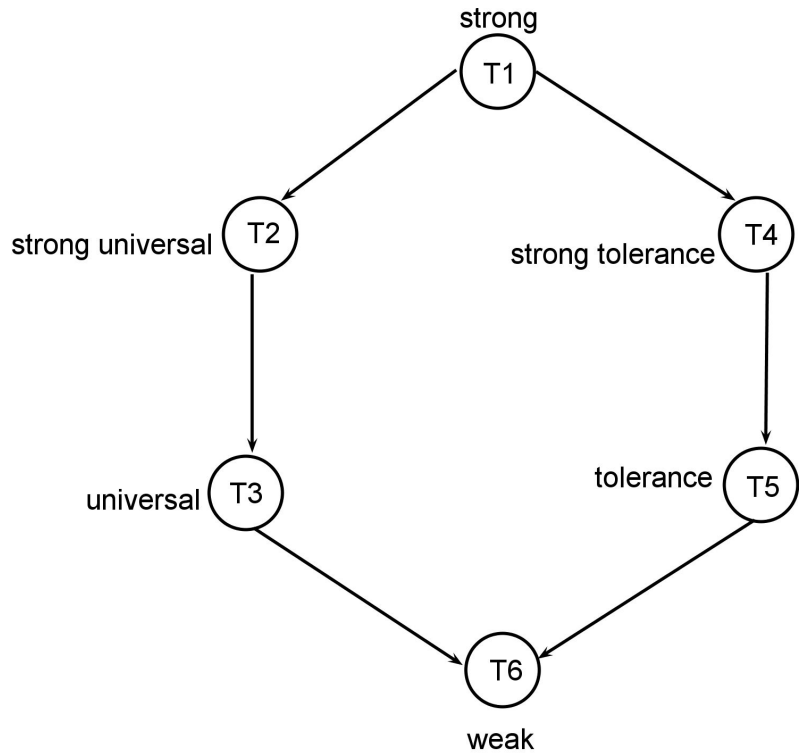

Fig. Hasse diagram of the relations between different types of monotone IFNSEvs.

The results from this section are summarized by the Hasse diagram in Figure where every arrow indicates an implication between corresponding types, and a non-existing arrow indicates that there is no implication between corresponding types of monotone IFNEvs.

\section{Conclusion}

In this paper we proposed six types of IFNSEvs of IFNSMs and the necessary and sufficient conditions are described. Also, we discussed the relations between these types of monotone FNSEvs and this results are summarized by the hasse diagram.

\section{References}

[1] Arockiarani. I, Sumathi. I. R., and Martina Jency, Fuzzy Neutrosophic Soft Topological Spaces, IJMA,4(10) (2013), 225-238.

[2] Arockiarani. I and Sumathi. I. R., A Fuzzy Neutrosophic Soft Matrix Approach in Decision making, JGRMA, 2(2) ,(2014), 14-23.

[3] K.Atanassov, Intuitionistic Fuzzy Sets, Fuzzy Sets and System, 20, (1983), 87-96.

[4] Broumi. S, Sahin. R, Smarandache. F., Generalized interval neutrosophic soft set and its decision making problem, Journal of New Results in Science, 7, 2014, 29-47.

[5] Bustince. H, Burillo. P., Vague sets are intuitionistic fuzzy sets , Fuzzy Sets and Systems, 79 (3), 1996, 403-405.

[6] Cechlarova. K., Eigenvectors in Bottleneck Algebra, Linear Algebra and its Application, 175(15), (1992), 63-73.

[7] Cechlarova. K ., Solution of Interval Linear Systems in (max, +)-algebra, In.Proc. 6th Internat. Symposium on Operational Research, Preddvor, Slovenia (2001), 321326.
[8] Cechlarova. K and Cuninghame-Green. R. A., Interval Systems of Max-Separable Linear Equations, Lin. Algebra Appl. 340, (2002), 215-224.

[9] Cuninghame-Green. R. A., Minimax Algebra, Lecture notes in Economics and Mathematics System 166, Springer-Verlag, Berlin, (1979).

[10] Fiedler. M, Nedoma. J, Ramik. J, Rohn. J and Zimmermann. K., Linear Optimization Problems with Inexact Data, Springer-Verlag, Berlin (2006).

[11] Gavalec. M., Monotone Eigenspace Structure in Max-Min Algebra, Linear Algebra and its Application, 345(1-3), (2002), 149-167.

[12] Gavalec. M, Plavka. J., Monotone Interval Eigenproblem in Max-Min Algebra, Kybernetika, 46(3), (2010), 387396.

[13] Gau. W. L., Buehrer. D. J, Vague sets, IEEE Trans. Sys Man Cybernet, 23(2), 1993, 610-614.

[14] Gavalec. M and Zimmermann. K., Classification of solutions to Systems of two-sided Equations with Interval Coefficients, Internat. I.Pure Apllied Math. 45 (2008), 533-542.

[15] Deli. I, Broumi. S., Neutrosophic Soft Sets and Neutrosophic Soft Matrices based on Decision Making, Journal of Intelligent and Fuzzy Systems, 28(5), (2014), 1-28.

[16] Kavitha. M, Murugadas. P and Sriram. S., Minimal Solution of Fuzzy Neutrosophic Soft Matrix, Journal of Linear and Topological Algebra, 6, (2017), 171-189.

[17] Kavitha. M, Murugadas. P and Sriram. S, On the $\lambda$ Robustness of Fuzzy Neutrosophic Soft Matrix, International Journal of Fuzzy Mathematical Archive, 2, (2017), 267-287.

[18] Kavitha. M, Murugadas. P and Sriram. S., Computing the Greatest X-eigenvector of Fuzzy Neutrosophic Soft Matrix, Internation Journal of Mathamatics and its Applications, 4, (2017), 893-907.

[19] Kavitha. M, Murugadas. P and Sriram. S., On the Power of Fuzzy Neutrosophic Soft Matrix, Journal of Linear and Topological Algebra, 7, (2018), 133-147.

[20] Kim. K.H, Roush. F.W., Generalized Fuzzy Matrices, Fuzzy Sets and System, 4 (3), 1980, 293-315.

[21] Maji .P. K., Biswas. R, and Roy. A. R., Fuzzy Soft Sets, The journal of Fuzzy Mathematics, 9(3), (2001), 589-602.

[22] Maji .P. K, Biswas. R, and Roy. A. R., An Application of Soft Sets in a Decision Making Problem, Computer and Mathematics with Applications, 44(8-9), (2002), 10771083.

[23] Maji. P. K., A Neutrosophic Soft Set Approach to a Decision Making Problem, Annauls Fuzzy Mathematics Information, 3(2), (2012), 313-319.

[24] Maji .P. K, Roy. A. R and Biswas. R., On Intuitionistic Fuzzy Soft Set, International Journal of Fuzzy Mathematics, 12(3), (2004), 669-684.

[25] Maji. P. K., Neutrosophic Soft Set, Annals of Fuzzy Mathematics and Informatics, 5(1), (2013), 157-168.

[26] Moore. R., Interval arithmatic, Prentice- Hall, Engle- 
wood Cliffs, NJ, USA.

[27] Pawlak. Z., Rough sets, International Journal of Information and Computer Sciences, 11 (5), 1982, 341-356.

[28] Manoj Bora, Banashree Bora, Tridiv Sut., Intuitionistic fuzzy soft matrix theory and its application in medical diagnosis, Annals of Fuzzy Mathematics and Informatics, x, 2013, pp. 1-xx.

[29] Molodtsov. D., Soft set theory first results, Computer \& Mathematics with Applications, 37 (4-5), 1999, 19-31.

[30] Rohn. J., Systems of Linear Interval equations, Lin. Algebra Appl, 126 (1989), 39-78.

[31] I.R. Sumathi and I.Arokiarani, New Operations on Fuzzy Neutrosophic Soft Matrices, International journal of innovative Research and Studies, 3(3), (2014), 110-124.

[32] Smarandache. F., Neutrosophy and neutrosophic logic, First International Conference on Neutrosophy, Neutrosophic Logic Set, Probability and Statistics University of New Mexico, Gallup, NM 87301, USA, 2002.

[33] Turksen. I. B., Interval valued fuzzy sets based on normal forms, Fuzzy Sets and Systems, 20 (2), 1986, 191-210.

[34] Thomason M.G., Convergence of Powers of fuzzy matrix, J. of Math. Anal and Appl, 57, 1977, 476-480.

[35] Smarandache. F., Neutrosophic set, a generalization of the intuitionistic fuzzy set, Inter. J.Pure Appl Math, 24 (3), 2005, 287-297.

[36] Sumathi. I.R and Arockiarani. I., New operations on fuzzy neutrosophic soft matrices, International Journal of Innovative Research and Studies, 3(12), 2014, 110-124.

[37] R.Uma, P.Murugadas and S.Sriram, Fuzzy Neutrosophic Soft Matrices of Type-I and Type-II, Communicated.

[38] L.A.Zadeh, Fuzzy Sets, Information and control, 8, (1965), 338-353.

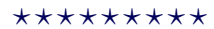

$\operatorname{ISSN}(\mathrm{P}): 2319-3786$

Malaya Journal of Matematik

ISSN(O):2321-5666
} 